\title{
An Automatic Multiple Sclerosis Lesion Segmentation Approach based on Cellular Learning Automata
}

\author{
A Case Study \\ Mohammad Moghadasi ${ }^{1}$, Dr. Gabor Fazekas ${ }^{2}$ \\ Department of Informatics \\ University of Debrecen, Debrecen, Hungary
}

\begin{abstract}
Multiple Sclerosis (MS) is a demyelinating nerve disease which for an unknown reason assumes that the immune system of the body is affected, and the immune cells begin to destroy the myelin sheath of nerve cells. In Pathology, the areas of the distributed demyelination are called lesions that are pathologic characteristics of the Multiple Sclerosis (MS) disease. In this research, the segmentation of the lesions from one another is studied by using gray scale features and the dimensions of the lesions. The brain Magnetic Resonance Imaging (MRI) images in three planes (T1, T2, PD) (weighted) containing MS disease lesions have been used. Cellular Learning Automata (CLA) is applied on the MRI images with a novel trial and error approach to set penalty and reward frames for each pixel. The images were analyzed in MATLAB and the results show the MS disease lesions in white and the brain anatomy in red on a black background. The proposed approach can be considered as a supplementary or superior method for other methods such as Graph Cuts (GC), fuzzy c-means, mean-shift, k-Nearest Neighbor (KNN), Support Vector Machines (SVM).
\end{abstract}

Keywords-Multiple Sclerosis (MS); MATLAB; Magnetic Resonance Imaging (MRI); MS Lesions; Cellular Learning Automata (CLA); Segmentation; Support Vector Machines (SVM)

\section{INTRODUCTION}

Multiple Sclerosis (MS) is a demyelinating nerve disease which for an unknown reason assumes that the immune system of the body is affected, and the immune cells begin to destroy the myelin sheath of nerve cells. In Pathology, the areas of the distributed demyelination are called lesions that are pathologic characteristics of the MS disease [1], [3].

Statistics show that over 2.5 million people in the world happen to suffer either of the stages of Multiple Sclerosis (MS). Fundamentally, the disease tends to erode the insulating myelin sheaths which surround the axons of the brain of an individual. It is correct to note that the disease happens to affect different people based on their anatomy. The statement implies that ounce a person has been diagnosed with MS then one may face the relapse and the remission periods at different rates. Plaque detection of $\mathrm{MS}$ can be perceived as a transformative step that has aided in spotting the type of MS and start the appropriate treatment during the early stages. The statement implies that having proper imaging techniques provides a clear visual analysis of the situations and thus the proper forms of medication can be administered during the early phases. Therefore, this research paper aims to assess the how MS detection has been enhanced and improved using the different forms of digital image processing. Multiple Sclerosis (MS) is a disorder which happens to affect the immune system of an individual and the traumatizing aspect is the fact that it eats or erodes the protective covering of the nerves. The disorder has many symptoms which are evident especially in the early phase because it affects the central nervous system. In this case, treating MS by making use of digital image processing is a technique which has enabled health practitioners to curb the effects of multiple sclerosis in individuals. It is correct to point out the evolution of digital imaging has provided the health practitioners an easier time in diagnosing the status of individuals and providing them with proper therapeutic channels. There are various issues which affect the functioning and the operations of the nerves and thus, the evolution of such technological mechanisms has been a major booster in coming up with appropriate ways of addressing the existing health issues. Examples of such disorders include Multiple Sclerosis.

Multiple Sclerosis (MS) cannot be diagnosed well by CT scan because the lesions are not clearly visible, but it is well diagnosed through MRI imaging. Since the immune cells around the blood vessels have more access to the nervous tissue, in MR imaging method, MS disease lesions are usually seen around the blood vessel. Because the MS disease lesions are only detectable by using MRI imaging, it has been employed in this research including T1, T2, and PD images (MICCAI Grand Challenge 2008 dataset) ${ }^{1}$, for MS lesions extraction [1], [3], [8]. Assessment of the proposed method is performed on the MICCAI MS Lesion Segmentation Challenge 2008 dataset.

The prevalent MRI species for the distinction of MS lesions are T1-weighted $^{2}$, T2-weighted ${ }^{3}$, Proton-density-weighted (PDweighted) $)^{4}$ fluid-attenuated inversion recovery(FLAIR). Unfortunately, the last one (FLAIR type) has a long acquisition

\footnotetext{
MICCAI is the annual international conference on Medical Image Computing and Computer Assisted Intervention

${ }^{2}$ T1-weighted (maximum T1 contrast shown)

${ }^{3} \mathrm{~T} 2$-weighted (maximum $\mathrm{T} 2$ contrast shown)

${ }^{4}$ Proton density (PD) weighting (density of hydrogen protons shown)
} 
time and may not be accessible for every patient [9]. Therefore, our proposed method is implemented by T1, T2, and PD image types.

In recent years, MS plaque detection based on color has attracted the attention of the many scholars for its low computational complexity. Most of these methods have a high error rate for not taking it into account the texture of the lesion. In this article, a novel method to detect MS lesions is proposed. This method combines the image information in the gray area and texture, detects the skin area by using Cellular Learning Automata (CLA) [10], [11], [12].

Cellular Learning Automata (CLA), the combination of Cellular Automata (CA) and Learning Automata (LA), is preferable to both CA and LA individually [4], [16]. Unlike CA or LA, CLA tries to learn optimal actions and it can improve the learning capability by using a set of learning automata that interact with each other. In addition, CLA can optimize outside standard sequential processes mapping real world solutions more accurately [2], [5], [16].

Firstly, the candidate lesion regions are detected through defining a threshold, and then these regions are assigned to a texture analyzing system. The output of texture analyzing system is the probability rate of being lesion for each pixel. Then this probable mapping is given to a CLA. By using neighborhood relations and texture information, CLA converges to a steady state representing the final output of the proposed system.

\section{BACKGROUND STUDIES}

Clustering according to the learning automata:

Clustering can be considered as the most important method in unsupervised learning. In clustering, the concern is to divide the data into clusters so that the similarity among the data of each cluster is at its maximum, and the similarity within the clusters is at its minimum. Clustering follows an iterative procedure that tries to estimate the followings for a considering number of constant clusters: obtaining some points as the centers of the clusters, that in fact these points are the means of the points belonging to each cluster. Assigning each sample data to a cluster where the data was closest to the center of the cluster [4], [12]. In the simplified type of this method, first, depending on the number of required clusters, some points are randomly chosen. Then, the data are assigned to one of the clusters according to their nearness (similarity), and thus new clusters are formed. By repeating this procedure, in every iteration, through obtaining the mean of the data, new centers can be calculated for the data, and they can be assigned to the new clusters again. This procedure would go on until no change would take place among the data. The following function (1) is known as target function [6], [10], [14], [15].

$$
j=\sum_{j=1}^{k} \sum_{i=1}^{x}\left\|x_{i}^{(\mathrm{j})}-c_{j}\right\|^{2}
$$

\section{Cellular Learning Automata (CLA)}

CLAs are a mathematical model for the systems that are dynamic, complex and consist of very simple components, which have learning capability, and behavior of each component is determined and corrected by the behavior of its neighbors and its past experiences. The simple components which form this model can show a complex and powerful behavior through interacting with one another [12], [13], [14].

Pixel based methods judge whether a pixel is a lesion pixel only by its color, while region based methods combines color and texture information [11]. Since using neighborhood and texture information can be practical in the skin detection, and since CLA can easily use neighborhood information [12], it can therefore be a suitable candidate to be employed for lesion detection. CLA is composed of the components that are in the same neighborhood and the behavior of these components is defined according to the current behavior of their neighbors and their own and their neighbors' experiences (reward and punishment that they receive for their actions and reinforcement signals that they receive from environment).

CLA is a hybrid model of two, learning automata and cellular automata. In the following these two models are discussed. Cellular automata are a mathematical model that can be used for the simulation calculations of the systems. Cellular automata are simple discrete systems that can exhibit complex calculations and behavior through simple and local rules. Locality in the cellular automata means that in determining the new value of each cell, the cells in its neighborhood are used and more distant cells would have no usage. Each cell has a set of states for itself and at every moment, according to its own state and the state of the neighbors, it decides to what state it goes. State switch rules are constant for cellular automata and never change. Cellular network can have different dimensions and be one, two or multi-dimensional [11]. According to the values that cells can possess, cellular automata are classified to two types of binary and multi-valued. Understanding the behavior of the cellular automata from its rules is very difficult and requires simulation. One of the problems of using cellular automata is designing rules that perform our desired action [13]. There are different types of cell updating rules that cause different types of cellular automata to be generated. For example, the rules can be expressed in probabilistic and deterministic forms and these two types of rules lead to two types of deterministic cellular automata and probabilistic cellular automata. Characteristics of the cellular automata can be briefly expressed as follows: automata are homogeneous and updating would be performed synchronously. Of course, asynchronous updating would also be possible, i.e. we start updating from one corner of the network and continue to the end, while in the synchronizing state, all the cells become updated according to the previous state of the neighbors not according to their present state. Dominant rules are defined according to the neighbors of each cell, and can have deterministic or probabilistic form. Learning automata are applied in cases such as physical processes simulations like Brownian motion, dissolution, social processes simulation like rumor dissemination, chemical phenomena simulation like fire spread and iron corrosion, image processing, random number generation, and cryptography [4], [10], [12]. Given its rules, 
learning automata can show complicated behaviors. Rules in the learning automata can be defined as a bit string in which each bit represents the next state corresponding to the number of the bit [2], [5].

\section{LEARNING AUTOMATA}

The process by which living creatures learn different subjects has for long time attracted the interest of the specialists. The research conducted on this ground focuses on two general categories: understanding the process through which living creatures attempt learning. Obtaining methods by which this learning ability can be created in the machines [11].

Learning is defined in different ways. Therefore, learning is defined as applying a series of modifications in the system efficiency, according to the past experiences. The most important characteristic of this learning system is its ability to increase efficiency during the time. To express this definition in a completely mathematical way, we should say: the objective of a learning system is to optimize an action that is not possible to be completely recognized. By this definition, the function of learning system can be reduced to a matter of optimization that is defined on a set of parameters, and can be solved by obtaining the set of optimal parameters through the existing methods. Learning automata method in learning is defined as determining the optimal action from among a limited set of predetermined actions that are capable of being performed in a random environment. Automata are assumed to work in an unknown random environment [11], [12]. At every moment it chooses an action from its action-set and notifies the environment. In response to the performed action, environment produces an output from the set of defined outputs (that can be limited or unlimited) and notifies the automata, and after receiving the response of the environment, the automata update their decision-making method for selecting next action. It is assumed that there is a probable relation between each automaton action and the response of the environment, and this relation is, in fact, the internal characteristics of the environment and is recognized by the automata during learning. Learning automata theories analyze the automata that work in such environment [14], [15].

Fig. 1 shows the relation between LA and the environment. An action randomly selected as a sample of action probability distribution. The selected action is then used in the environment [17]. The environment responds to the taken action in turn with a signal called reinforcement signal. Afterward, the action probability vector is updated based on the reinforcement signal feedback from the environment.

In the 1960s, a method was introduced to simplify the existing problems in the engineering systems to a problem of identifying optimal parameters and applying hill climbing methods to solve it. Learning automata that worked in a random environment is considered as a model for learning. In the conducted researches, using learning in the engineering systems was also considered.

a) Irregular Cellular Learning Automata (ICLA): Irregular cellular learning automata (ICLA) are cellular learning automata (CLA) which remove the restriction of rectangular grid structure in traditional CLA. There are number of applications which cannot be adequately modeled with rectangular grids. Each pixel represents a cell that is equipped with a learning automaton. Learning automata are in fact a specific cell that determines its state according to action probability vector [7], [11], [14].

b) Proposed algorithm: In many matters, learning cannot be correctly operated by using one learning automaton, but the learning automata manifest their main power when employed collectively. Given this issue and the defects enumerated for cellular automata, by combining these two models, a new model called cellular learning automata was generated. To use the algorithm, we first need to have an initial image from the location of the MS plaque (Fig. 6). The main objective of employing cellular learning automata is making use of neighborhood relations among the pixels of pseudo-skin regions to increase the efficiency [10], [14], [18]. This is because a pixel doesn't necessarily denote the concept of being a plaque by itself, and a set of pixels can define a skin region in a better way (Fig. 2 to 5). The penalty and reward of each cell are according to the action chosen by the central automata and its neighbors. If the number of the cells that choose the appropriate action for plaque detection is more than 7 , the central automata will be rewarded. Therefore, if seven automata detect a texture as plaque, the region around the central automata is taken as plaque region. If the number of these automata is less than 4 (Fig. 4 and 5), the central automata will be penalized [12], [13].

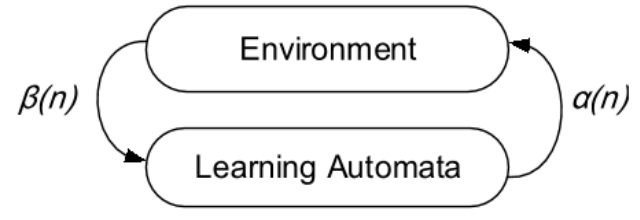

Fig. 1. Interaction between Learning Automata and the Environment [15].

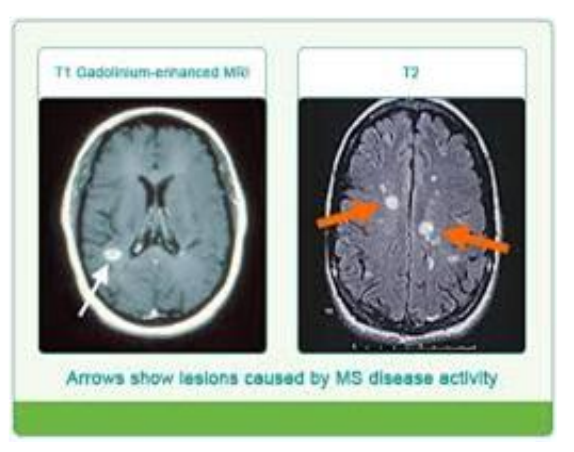

Fig. 2. MS Disease Lesions.

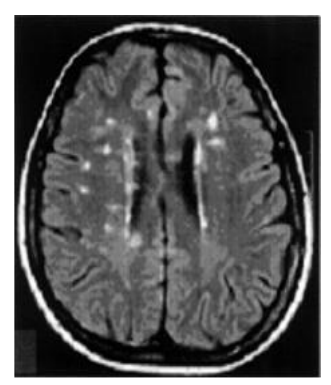

Fig. 3. Image Sample of MS. 


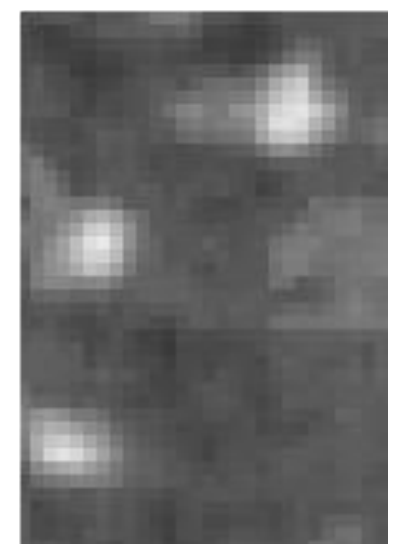

Fig. 4. Infected Sample.

The output result will be as below:

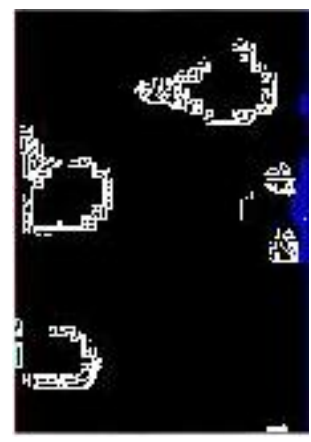

Fig. 5. The resulted image in MATLAB

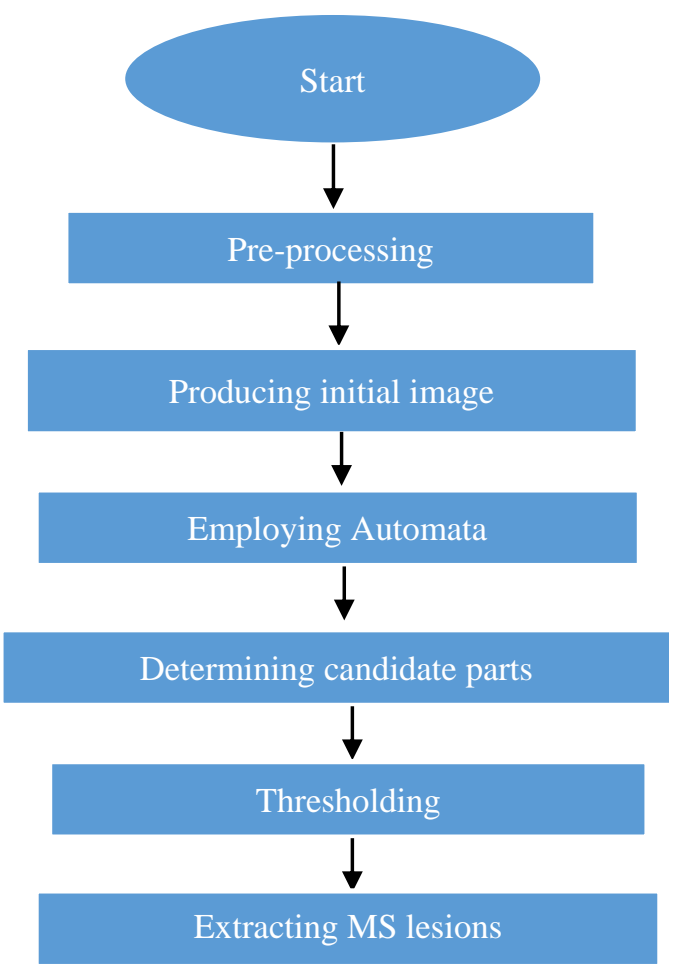

Fig. 6. Block Diagram Illustrating the Proposed Algorithm.
The proposed algorithm

The Pseudo algorithm of the proposed algorithm is as follows:

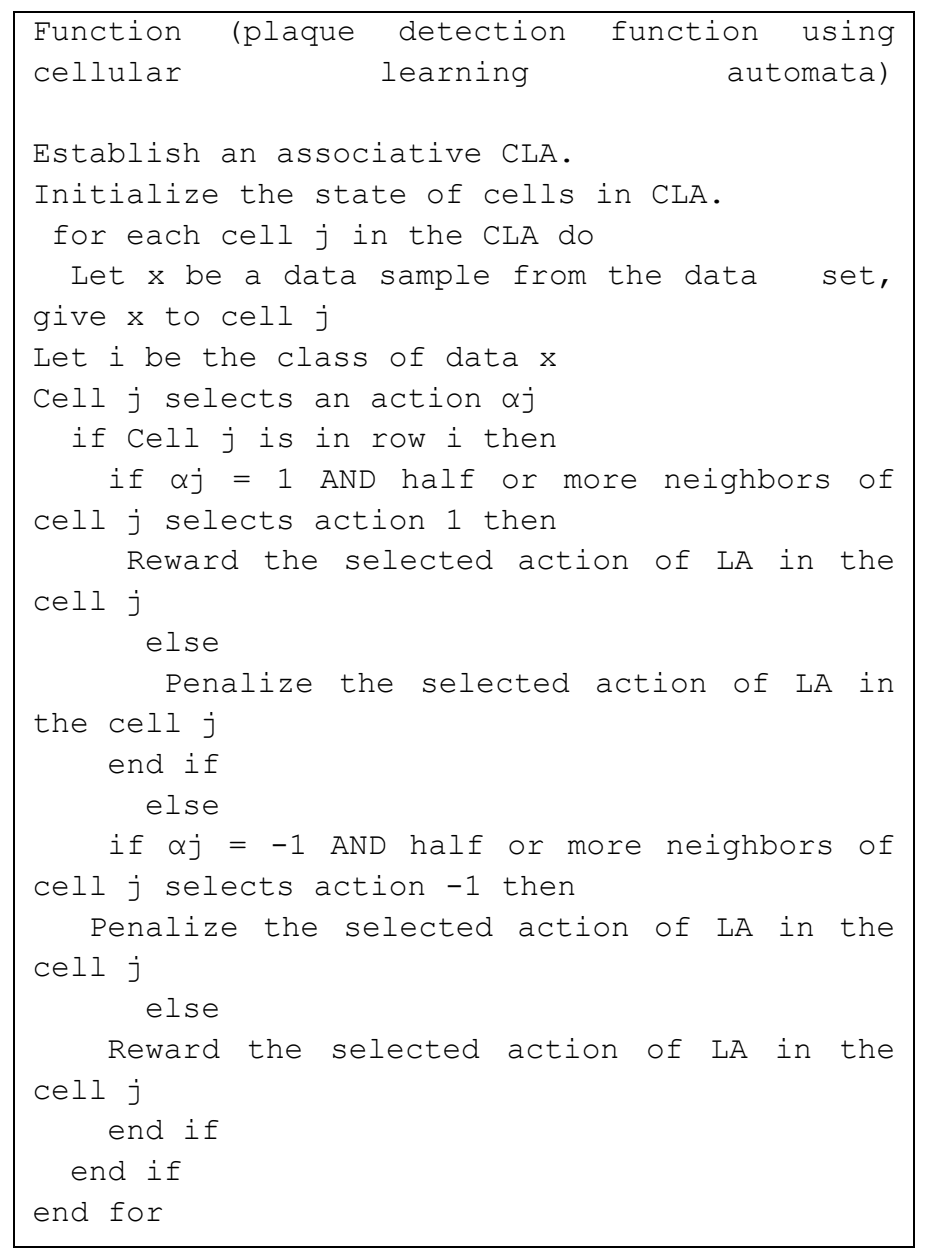

\section{RESUlTS AND CONCLUSION}

Using databases of the MS images, the images are chosen that have desirable condition regarding the plaques information. For the rate of detection, a formula is used to calculate the percentage error: pixels mistakenly detected as plaques to the total percentage of the detected pixels (Fig. 5). The aforementioned-phrase is the assessment factor for the percentage error (Fig. 7), (Table I), and this formula was examined in 40 images of the database and on average, the percentage error is as follows.

\section{TABLE. I. ANALYZED IMAGES}

\begin{tabular}{|l|l|l|}
\hline $\begin{array}{l}\text { Number of the } \\
\text { analyzed images }\end{array}$ & $\begin{array}{l}\text { Error rate in finding the } \\
\text { pixels that were } \\
\text { mistakenly detected as } \\
\text { plaques }\end{array}$ & $\begin{array}{l}\text { Error rate in finding the } \\
\text { pixels that mistakenly } \\
\text { weren't detected as } \\
\text { plaques }\end{array}$ \\
\hline 40 images & $10 \%$ & $7 \%$ \\
\hline
\end{tabular}




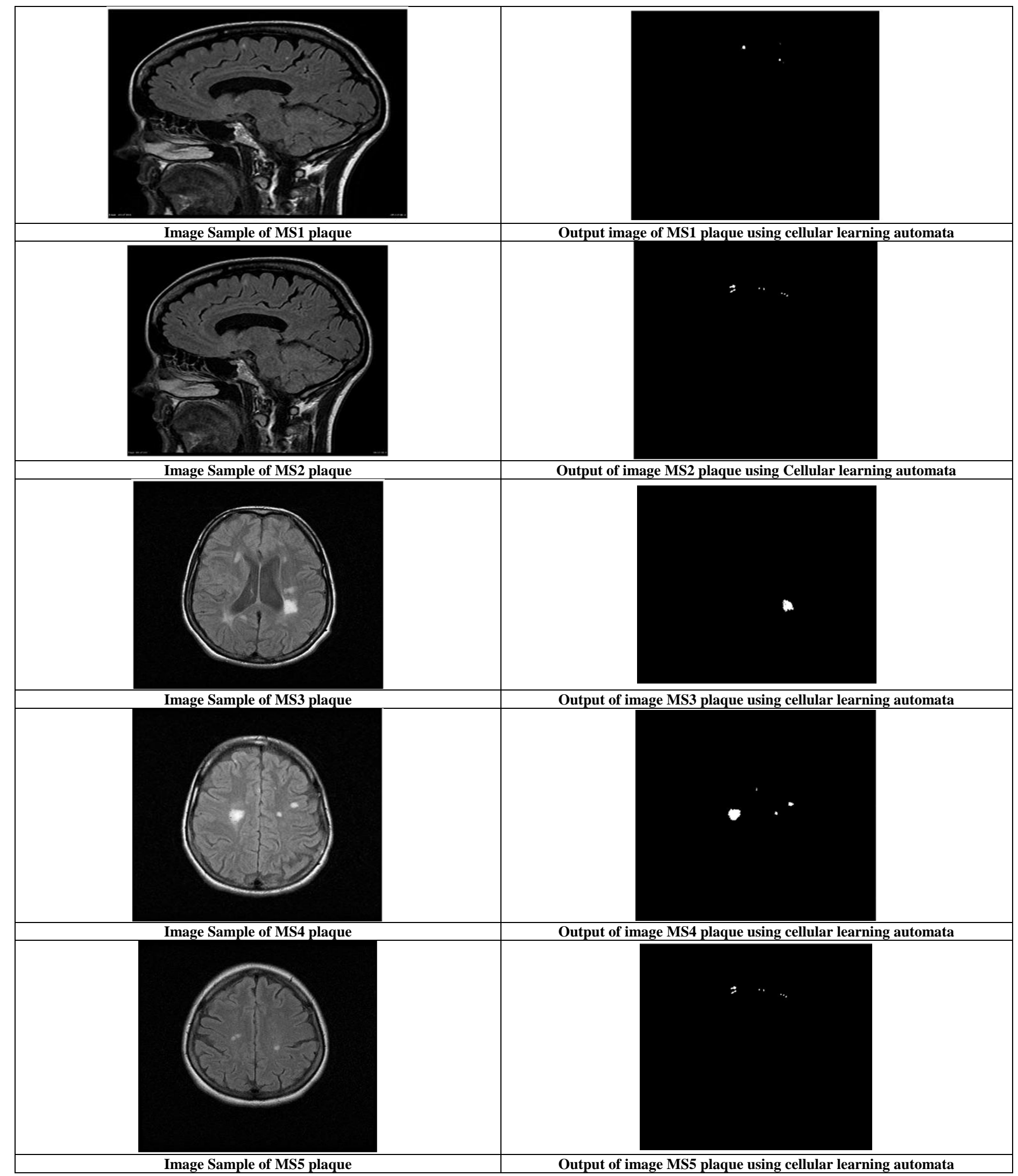

Fig. 7. MS Images-Analyzed Images. 
The pixels depicting MS plaque can be found in the image. By visual inspection, the plaque regions are specified by 1 and other regions are specified by 0 . Using ROC command in MATLAB, ROC curve is plotted which is as it is presented below. This curve is used to compare two classification methods. Its Vertical axis is the true positive rate (TPR) and its horizontal axis is the false positive rate (FPR).

This curve represents the tradeoff between TPR and FPR for the two-classed classification of MS and healthy plaques. To plot the curve, we assume a threshold; values above the threshold will be considered as "yes" and values below "No".

Conclusively, it is evident that there are various techniques which can be applied in MS plaque detection using the various imaging aspects. From the research paper, it is clearly obvious that there are various mechanical and operational advantages and disadvantages which emanate from the use of the systems. In addition, the health practitioners need to have a working knowledge of the different systems so that they can be in different positions to adequately deliver as per the required levels. Each technique has its specific advantage and disadvantage and thus, as a health practitioner one need to affirm the best mechanism to set in place based on the gravity of the situation. Primarily, all these aspects can be used in a health institution and it is recommendable for one to have enough background knowledge of the patient before deciding to embark on a specific venture.

\section{FUTURE WORK}

This case study is limited to an existing database and university, and it reflected the result of a certain set of images in a known repository. In the next paper, near future, the SVM process and techniques can be conducted on a larger scale of images to determine factors influencing current results and lacks using SVM. This will help to understand the efficiency of these techniques and may lead to better results.

\section{REFERENCES}

[1] Fauci, A.S., Harrison's principles of internal medicine. Vol. 2. 2008: McGraw-Hill, Medical Publishing Division.

[2] Book by David B. Fogel, Derong Liu, and James M. Keller, "Fundamentals of Computational Intelligence: Neural Networks, Fuzzy Systems, and Evolutionary Computation", July 2016.

[3] Robert J. Fox. a,b Erik Beall. c Pallab Bhattacharyya. c Jacqueline Chen. $\mathrm{d}$ and Ken Sakaie. "Advanced MRI in Multiple Sclerosis: Current Status and Future Challenges" Neurologic Clinics, Elsevier, Volume 29, Issue 2, May 2011, Pages 357-380. doi: 10.1016/j.ncl.2010.12.011.
[4] Stephen Boyd, Neal Parikh, Eric Chu, Borja Peleato and Jonathan Eckstein (2011), "Distributed Optimization and Statistical Learning via the Alternating Direction Method of Multipliers", Foundations and Trends® in Machine Learning: Vol. 3: No. 1, pp 1-122. http://dx.doi.org/10.1561/2200000016.

[5] Baba, Abdellatif. 2013. "An introduction to neural networks" (1). 10.13140/RG.2.2.16523.69923.

[6] Nascimento, S., B. Mirkin, and F. Moura-Pires. A fuzzy clustering model of data and fuzzy c-means. in Fuzzy Systems, 2000. FUZZ IEEE 2000. The Ninth IEEE International Conference on. 2000. IEEE.

[7] Thomas A. Woolsey, Joseph Hanaway, Mokhtar H. Gado, The Brain Atlas: A Visual Guide to the Human Central Nervous System 3rd Edition, Publisher: Wiley-Liss; 3 edition 2007 December, ISBN-13: 9780470084762

[8] Woodward, P., MRI for Technologists. 2001: McGraw Hill Professional.

[9] Biediger, D., C. Collet, and J.P. Armspach, Multiple sclerosis lesion detection with local multimodal Markovian analysis and cellular automata 'GrowCut'. Journal of Computational Surgery, 2014. 1(1): p. 115.

[10] Abin, A.A., M. Fotouhi, and S. Kasaei, A new dynamic cellular learning automata-based skin detector. Multimedia systems, 2009. 15(5): p. 309323.

[11] Abin, A.A., M. Fotouhi, and S. Kasaei. Skin segmentation based on cellular learning automata. in Proceedings of the 6th international conference on advances in mobile computing and multimedia. 2008. ACM.

[12] Beigy, H. and M.R. Meybodi, Cellular learning automata with multiple learning automata in each cell and its applications. IEEE Transactions on Systems, Man, and Cybernetics, Part B (Cybernetics), 2010. 40(1): p. 5465.

[13] Beigy, H. and M.R. Meybodi, A mathematical framework for cellular learning automata. Advances in Complex Systems, 2004. 7(03n04): p. 295-319.

[14] Beigy, H. and M.R. Meybodi, Open synchronous cellular learning automata. Advances in Complex Systems, 2007. 10(04): p. 527-556.

[15] Vahidipour, S.M., M.R. Meybodi, and M. Esnaashari, Learning automata-based adaptive Petri net and its application to priority assignment in queuing systems with unknown parameters. IEEE Transactions on Systems, Man, and Cybernetics: Systems, 2015. 45(10): p. 1373-1384.

[16] Moradabadi. B, Meybodi. M.R, "Wavefront cellular learning automata", Chaos: An Interdisciplinary Journal of Nonlinear Science, 2018 Volume 28, Issue 2, https://doi.org/10.1063/1.5017852.

[17] Book chapter by A. H. Fathy Navid, A. B. Aghababa, Chapter 5, "Cellular Learning Automata and Its Applications", IntechOpen Book, "Emerging Applications of Cellular Automata" Edited by Alejandro Salcido, Published: May 8th, 2013, DOI: 10.5772/52953.

[18] Ahangaran, M., Taghizadeh, N., \& Beigy, H. (2017). "Associative cellular learning automata and its applications". Applied Soft Computing, 53, 1-18.doi:10.1016/j.asoc.2016.12.006. 